\title{
3D PRINTED MICROFLUIDIC SELECTABLE RATIO MIXER PUMP IN 2 MM $^{3}$ G.P. Nordin*, H. Gong, and A.T. Woolley \\ Brigham Young University, Provo, Utah, USA
}

\begin{abstract}
We report the use of $3 \mathrm{D}$ printing to fabricate a selectable ratio mixer pump in a volume of only $2 \mathrm{~mm}^{2}$. Our custom 3D printer and material is used to make very compact individual elements such as fluid channels, valves, and pumps that are laid out in $3 \mathrm{D}$ to achieve small overall device volume. The device includes two pumps, each connected to its own fluid source, and a two-chamber mixing unit that both mixes the fluid pumped into it from each fluid source, and acts itself as a pump to expel the mixed fluid.
\end{abstract}

\section{INTRODUCTION}

Our group has focused on developing 3D printing tools and materials for fabrication of microfluidic devices. To date, the key inhibiting factor for 3D printing has been the inability of commercial 3D printers and materials to fabricate truly microfluidic $(\leq 100 \mu \mathrm{m})$ structures rather than millifluidic structures [1]. The critical characteristic of microfluidic devices is that they consist primarily of a series of small, interconnected voids inside a bulk material. Such voids form a variety of necessary structures that include passive components (e.g., flow channels, splitters, mixers, reaction chambers, and droplet generators) and active components such as valves and pumps. Since the requirement for small, interlinked voids is in direct contrast to standard (non-microfluidic) 3D printing applications in which external features or sparse structures tend to be important, the vast majority of 3D printing technology development has been directed toward different goals than those required for successful printing of microfluidic devices.

We recently developed a custom stereolithographic 3D printer and a new photopolymerizable resin that enables the fabrication of flow channels with cross section as small as $18 \times 20 \mu \mathrm{m}^{2}$, which is a factor of $\sim 200$ smaller than the best that has been achieved with commercial tools and materials [2]. In this presentation, we demonstrate how the new printer and resin enable the realization of extremely small membrane valves and pumps, and focus particularly on the integration of such components in a dense 3D layout to realize a combination selectable ratio mixer and pump in a volume of only $2 \mathrm{~mm}^{3}$ (i.e., the size of a sesame seed). This device is an example of what can be achieved with purpose-built tools and associated custom materials for 3D printing of microfluidics.

\section{D PRINTED VALVES AND PUMPS}

In Ref. 3 we demonstrated and characterized 3D printed microfluidic valves and pumps using a commercial 3D printer and custom resin formulation, resulting in membrane valves (Figs. 1(a) and 1(b)) $1.08 \mathrm{~mm}$ in diameter. With our new custom 3D printer and material, we can substantially reduce valve and pump size. For example, a single fabricated valve is shown in Fig. 1(c) and a test array of 45 valves in Fig. 1(d) [4]. The valve diameters are 300 $\mu \mathrm{m}$. Each row of valves in the array has been tested to 10,000 actuations, except for the bottom row, which was tested to $1,000,000$ actuations. In all cases, there was no observable degradation in performance of the valves. Control and fluid channels are routed to the valves through compact chip-to-chip interconnects that are directly $3 \mathrm{D}$ printed as part of the device. We have demonstrated 400 interconnects at a density of $53 / \mathrm{mm}^{2}$ [4].
We have recently reduced the size of 3D printed valves even further $(150 \mu \mathrm{m}$ diameter), which results in valves that occupy a factor of 130x smaller volume compared to our work in Ref. 3.

Pumps consist of two valves and a valve-like structure, and are operated with a 5-phase cycle. Using the smaller valves fabricated with our custom $3 \mathrm{D}$ printer and resin, pump size is commensurately reduced.

\section{SELECTABLE RATIO MIXER PUMP}

The decrease in component size can be used to create extremely compact devices. As an example, consider the combination mixer and pump in Fig. 2, which features the ability to mix selectable ratios of two fluids. A block diagram is shown in Fig. 2(a), with a corresponding 3D CAD layout in Fig. 2(b). A microscope photo of a finished device is shown in Fig. 2(c), where the square shapes around the periphery are controlled compression integrated microgaskets (CCIMs), as described in Ref. [4], to make chip-to-chip interconnects to an interface chip.

To test operation of the device, a dye solution is mixed with water and video of the mixing process is analyzed. An example video analysis for 4 units of dye combined with 2 units of water is shown in Fig. 3. Based on a new mathematical model we have developed to guide our video analysis, the blue curve is the relative dye concentration in the output channel. After approximately 9 mixer-pump cycles, the relative dye concentration asymptotes to 0.71 , compared to an ideal value of 0.67 .

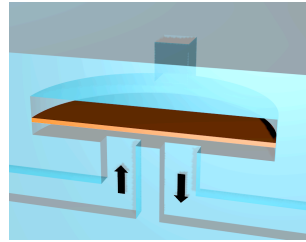

(a)

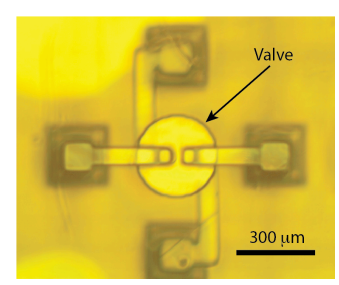

(c)

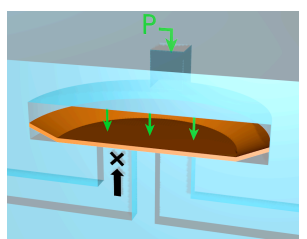

(b)

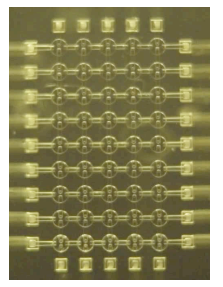

(d)
Figure 1: Schematic illustration of a pneumatic membrane valve open (a) and closed (b). (c) Microscope photo of a $3 D$ printed membrane valve and (d) an array of $45300 \mu \mathrm{m}$ diameter valves in a test configuration.

The measured relative dye concentration as a function of designed dye concentration is shown in Fig. 4(a). Using our new image analysis method, we can estimate the uniformity of the concentration across the outlet channel for each video frame. The smaller the standard deviation of the concentration within a frame, the better the fluid is mixed. The frame-to-frame standard deviation of the in-frame standard deviation is a measure of the degree of mixing over time, which is shown in Fig. 4(b). The 
results indicate that 4 to 5 mixing periods in the active mixer is sufficient to reach the noise floor of the measurement method.

\section{CONCLUSIONS}

We have demonstrated that 3D printing with our custom $3 \mathrm{D}$ printer and material can realize compact functional elements in a 3D (as opposed to planar) geometry that would be very difficult or impossible to fabricate with traditional methods.

\section{REFERENCES}

[1] MJ Beauchamp, GP Nordin, and AT Woolley, "Moving from millifluidic to truly microfluidic sub-100-mum cross-section 3D printed devices", Anal Bioanal Chem. 409, 4311 (2017).
[2] H Gong, BP Bickham, AT Woolley, and GP Nordin, "Custom 3D printer and resin for 18 um x 20 um microfluidic flow channels", Lab Chip. 17, 2899 (2017).

[3] H Gong, AT Woolley, and GP Nordin, "High density 3D printed microfluidic valves, pumps, and multiplexers", Lab Chip. 16, 2450 (2016).

[4] H Gong, AT. Woolley, and GP. Nordin, "3D printed high density, reversible, chip-to-chip microfluidic interconnects," Lab on a Chip, 18, 639-647 (2018).

\section{CONTACT}

*G.P. Nordin, tel: +1-801-422-1863; nordin@byu.edu

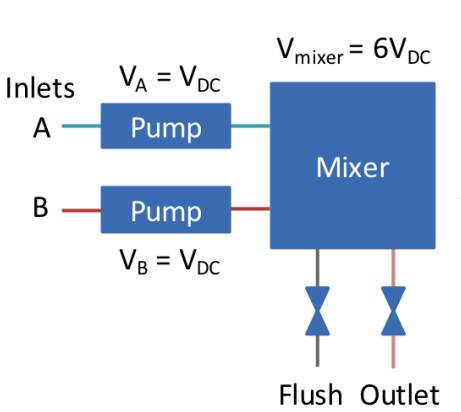

(a)

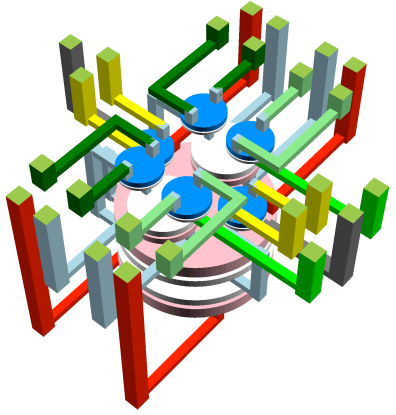

(b)

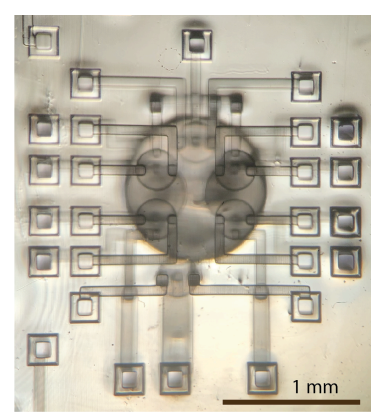

(c)

Figure 2: (a) Functional illustration of mixer-pump. (Vxx are volumes.) (b) 3D CAD layout with chip-to-chip interconnects. (c) Microscope photograph of $3 D$ printed mixer-pump and chip-to-chip interconnects. The volume occupied by only the mixer-pump itself is $1.2 \mathrm{~mm} x 1.5$ $m m \times 1.1 \mathrm{~mm}=2 \mathrm{~mm}^{3}$.

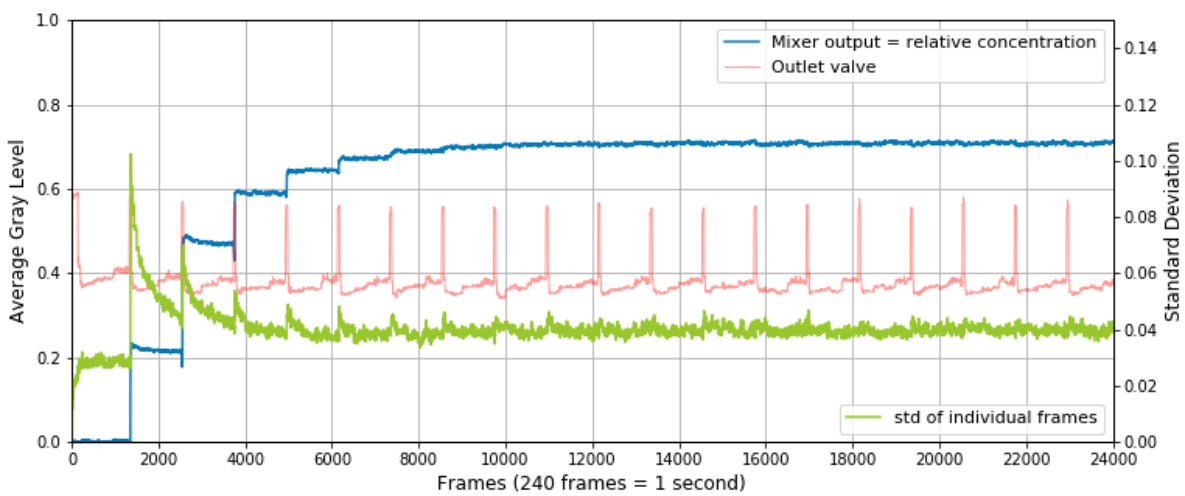

Figure 3: Result of processing video of mixer pump during operation. Blue-relative concentration, Green-standard deviation of relative concentration within each video frame, Red-outlet valve opens during short peaks.

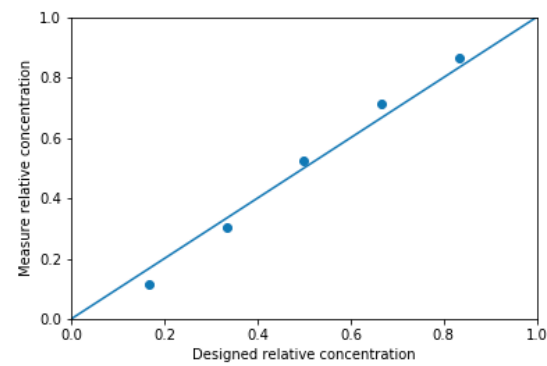

(a)

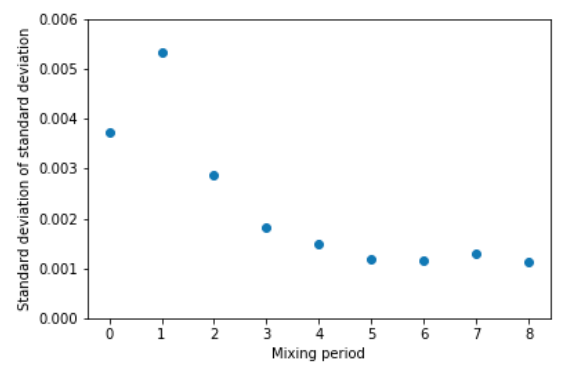

(b)

Figure 4: (a) Measured relative concentration as a function of designed relative concentration. Circles are measurements, line is ideal relationship. (b) Frame-to-frame variation of the in-frame concentration variation as a function of how many periods of back-and-forth fluid flow is performed in the mixer structure. 